\title{
A Look at the AP2 Beamline
}

\author{
Pbar note \#647 \\ Keith Gollwitzer \\ January 8, 2001
}

Some recent work has been done to look at improvements of transporting beam from the Lithium Lens to the Debuncher. This work has been done using the beamline modeling tools developed by Dave McGinnis. These tools, console application P143 and optimization code running MAD repeatedly on the Beam Physics UNIX system, were first used to match the Twiss and dispersion parameters at the end of AP2 to the Debuncher. Imaginary trims were then added to AP2 to study where additional trims could be used to help with beam control in small aperture areas.

\section{AP2-Debuncher Matching}

The AP2 beamline has been changed to match as well as possible the Debuncher parameters at the downstream end of the injection septum. The Debuncher Twiss values are in the following table and are from the console application P144.

\begin{tabular}{|c|c|c|}
\hline Parameter & Horizontal & Vertical \\
\hline$\beta$ & $3.93 \mathrm{~m}$ & $13.5 \mathrm{~m}$ \\
\hline$\alpha$ & 0.865 & -2.03 \\
\hline $\mathrm{D}$ & $0.0 \mathrm{~m}$ & $0.0 \mathrm{~m}$ \\
\hline $\mathrm{D}$ & 0.0 & 0.0 \\
\hline
\end{tabular}

Other goals of the optimization procedure included keeping the maximum dispersion and beam width smaller than $2 \mathrm{~m}$ and $0.09 \mathrm{~m}$, respectively. The attempt at matching the parameters is really a minimization of the emittance blowup. Phase space matching errors contributing to emittance changes are discussed in pbar note \#639. For clarity, equation 18 from that note should be written as

$$
\begin{aligned}
\varepsilon_{2}= & \frac{1}{2}\left[\frac{\beta_{1}}{\beta_{2}}+\frac{\beta_{2}}{\beta_{1}}+\frac{\left(\alpha_{2} \beta_{1}-\alpha_{1} \beta_{2}\right)^{2}}{\beta_{1} \beta_{2}}\right] \varepsilon_{1}+3\left[\beta_{2}(\Delta \theta)^{2}+2 \alpha_{2}(\Delta \theta)(\Delta x)+\gamma_{2}(\Delta x)^{2}\right]+ \\
& 3\left[\beta_{2}\left(\Delta D^{\prime}\right)^{2}+2 \alpha_{2}\left(\Delta D^{\prime}\right)(\Delta D)+\gamma_{2}(\Delta D)^{2}\right] \times\left(\frac{\sigma_{p}^{2}+(\Delta p)^{2}}{p_{2}^{2}}\right) .
\end{aligned}
$$

The subscripts 1 and 2 respectively denote the beamline and storage ring parameters. All $(\Delta \mathrm{K})$ quantities are absolute differences: $\left|\mathrm{K}_{1}-\mathrm{K}_{2}\right|$. The three terms are respectively Twiss parameter, injection steering and dispersion mismatches. If the $\Delta \mathrm{X}$ quantities are not treated as absolute differences, then the cross terms for the injection steering and dispersion could lessen the blowup depending up the sign of $\left(K_{1}-K_{2}\right)$. However, one can note that having the Twiss parameter $\alpha_{2}$ negative is desirable.

The initial parameters of the AP2 line, exiting the Lithium Lens, have been taken to be

$$
\begin{array}{ll}
\beta_{x}=\beta_{y}=4.5 m & \alpha_{x}=\alpha_{y}=0.0 \\
D_{x}=D_{y}=0.0 m & D_{x}^{\prime}=D_{y}^{\prime}=0.0
\end{array}
$$

In addition, both emittances are taken to be $24 \pi \mathrm{mm}$-mrad and the momentum spread to be selected by PMAG as $4 \%$. The optimizing code running MAD repeatedly on the Beam Physics system takes less than two hours to settle on a solution which has the emittances blowup 5\%. A set of optimized settings is given in the following table (all values are in Amps).

\begin{tabular}{|rr|rr|rr|rr|}
\hline Q701 & 362.55 & Q719 & 175.15 & QS719 & 0.00 & QS729 & 12.34 \\
\hline Q702 & 306.43 & Q729 & 395.25 & QS720 & 0.00 & QS730 & 28.30 \\
\hline Q707 & 109.90 & Q731 & 357.73 & QS722 & 4.82 & QS731 & 15.18 \\
\hline Q715 & 30.69 & & & QS723 & 20.00 & QS732 & 20.00 \\
\hline Q716 & 97.73 & QS716 & 10.12 & QS726 & 7.91 & QS733 & 2.06 \\
\hline Q718 & 215.20 & QS717 & 5.16 & QS728 & 46.65 & & \\
\hline
\end{tabular}


There is obvious work yet to be done by looking at the shunts that are at maximum and minimum values and seeing if starting the bus currents appropriately allows for more flexibility. Loading these settings and asking P143 to calculate the AP2 lattice, the lattice then can be displayed using P143.

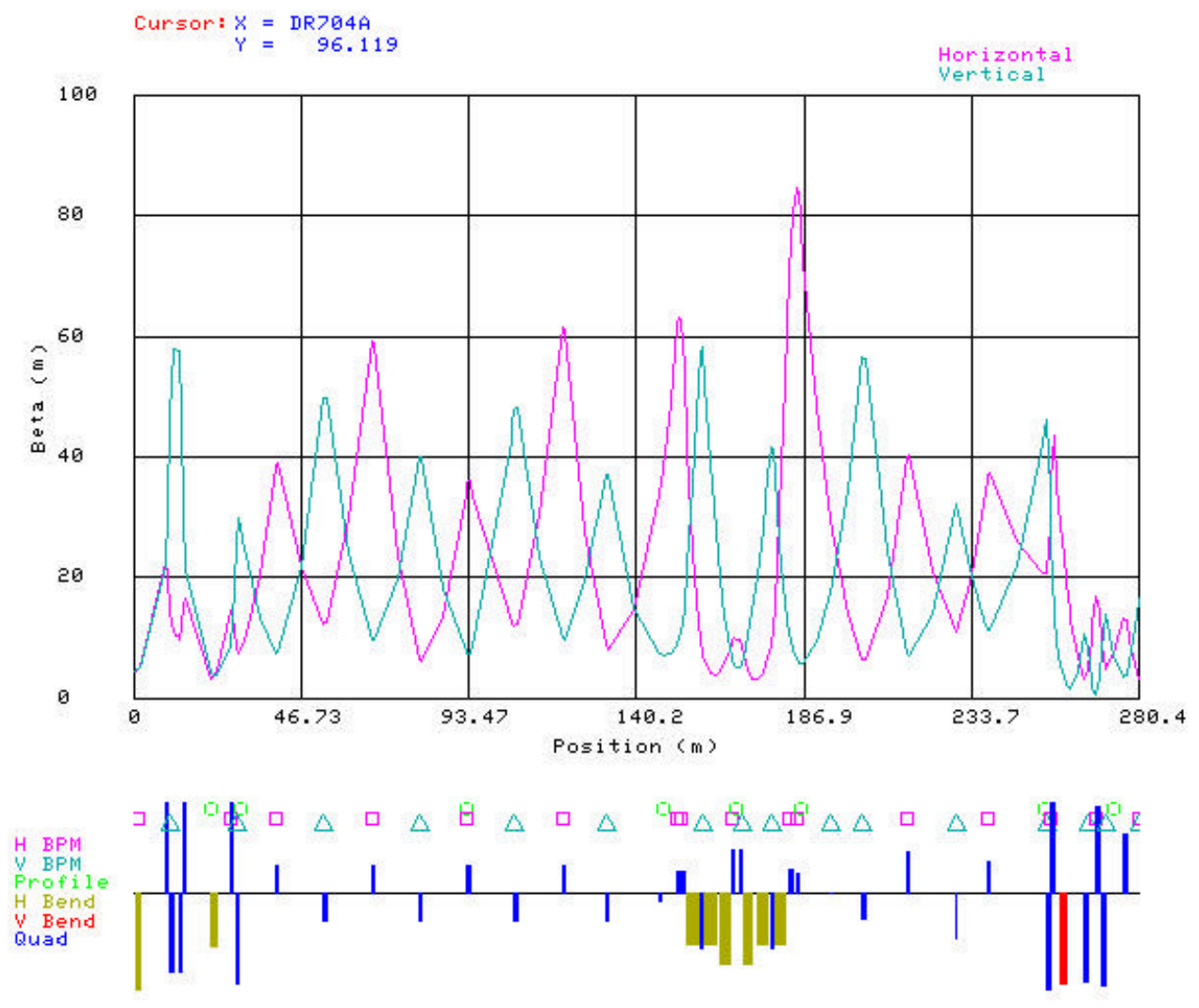

The beta functions are displayed in the above figure. The dispersion functions are shown below.

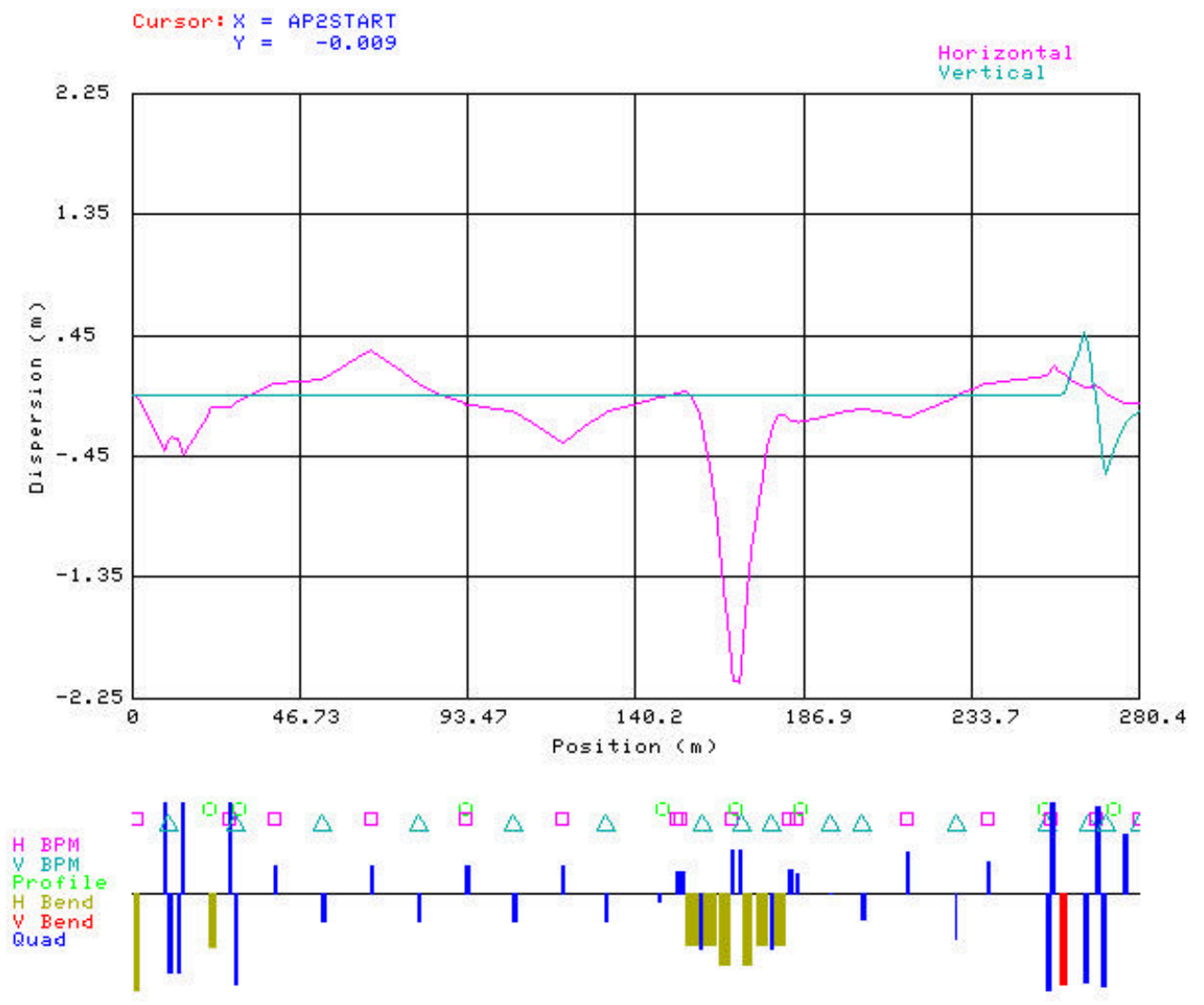




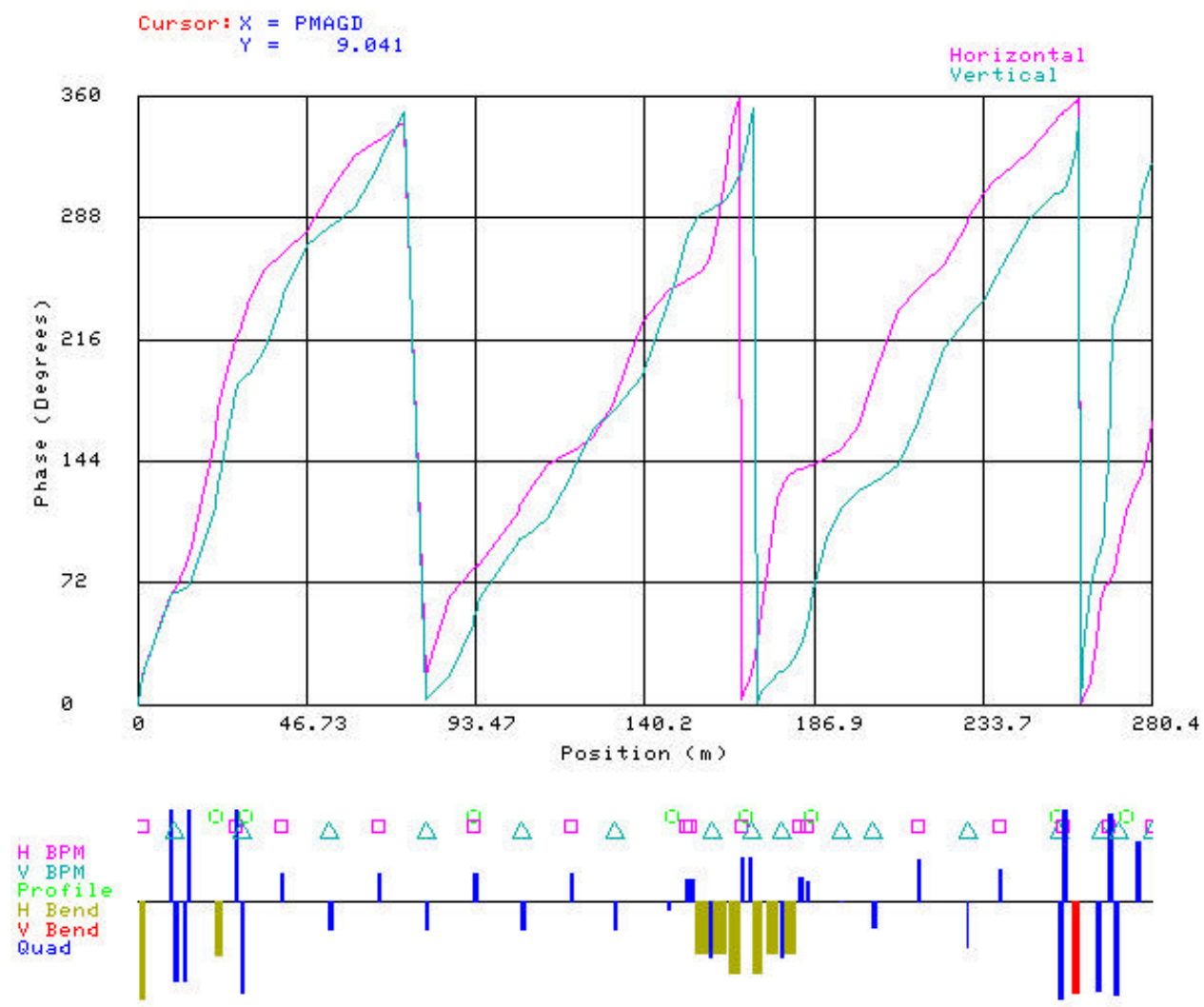

The phase advances are shown above while the transverse beam widths are shown below.

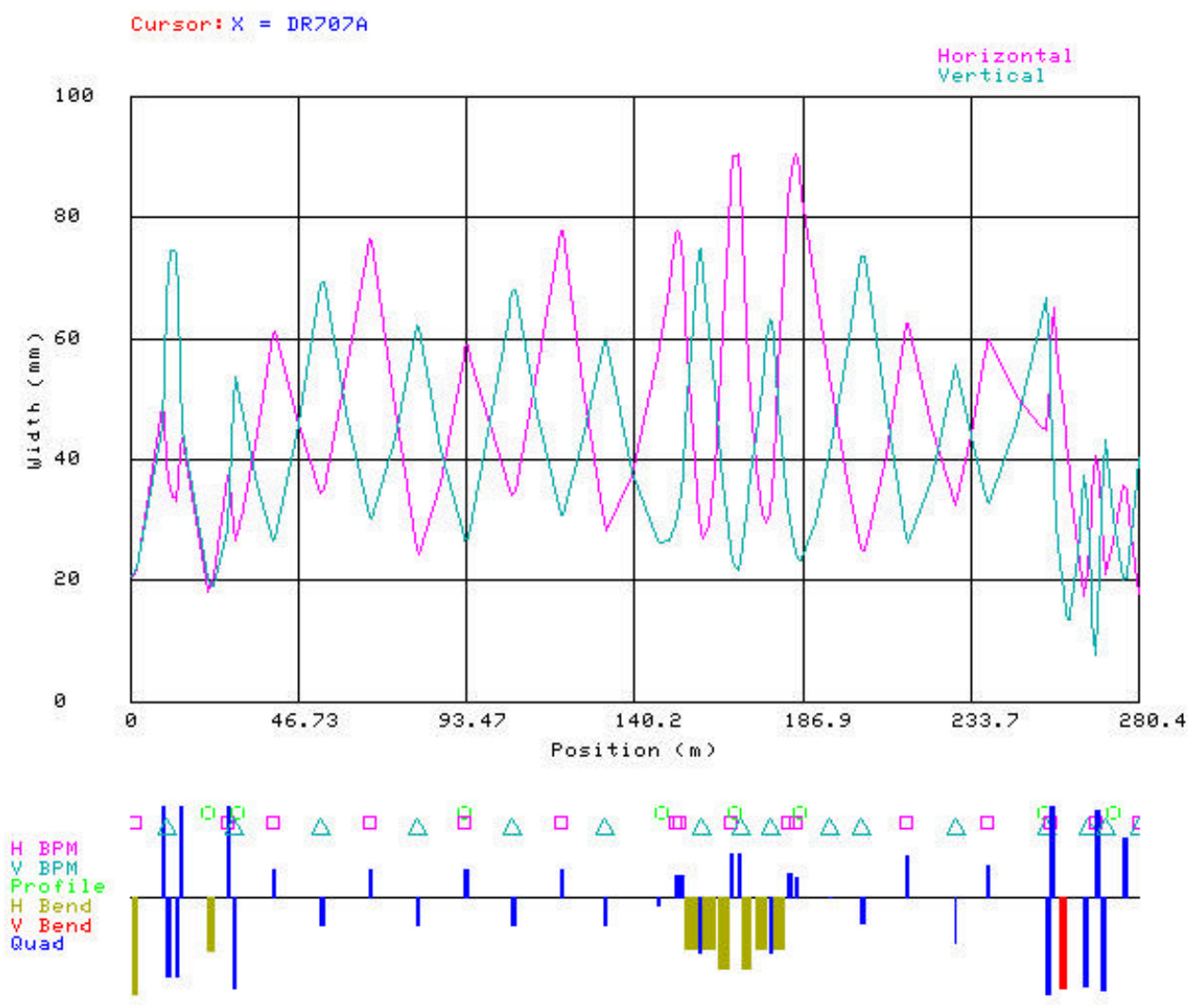




\section{AP2 Orbit Control}

The lattice files that P143 reads were copied and modified. Imaginary trims in both planes were put in the beamline whenever there was at least $1 \mathrm{~m}$ gap between elements of the lattice. These trim strengths were taken to be the same as HT730 and HT731 (the "weakest" beamline trim). P143 then read these modified files and the orbit correction part of the program was used to make bumps (BPM positions were set and then the correction routine was run). Nominally, to make local bumps anywhere in AP2, trims were needed every other quad due to the phase advance. More in depth studies were done at the injection area (here a fictitious BPM was added to the end of the AP2 line) and vertically at the left bend where the beam pipe is narrow compared to the rest of the line.

\section{Injection Region}

The phase advances near the end of the AP2 line are fairly quick. The phase advances over the last $60 \mathrm{~m}$ of the AP2 line are shown below. The positions of existing and proposed trims (labeled in bold) are indicated on the element bar as well as on the individual phase advance lines.

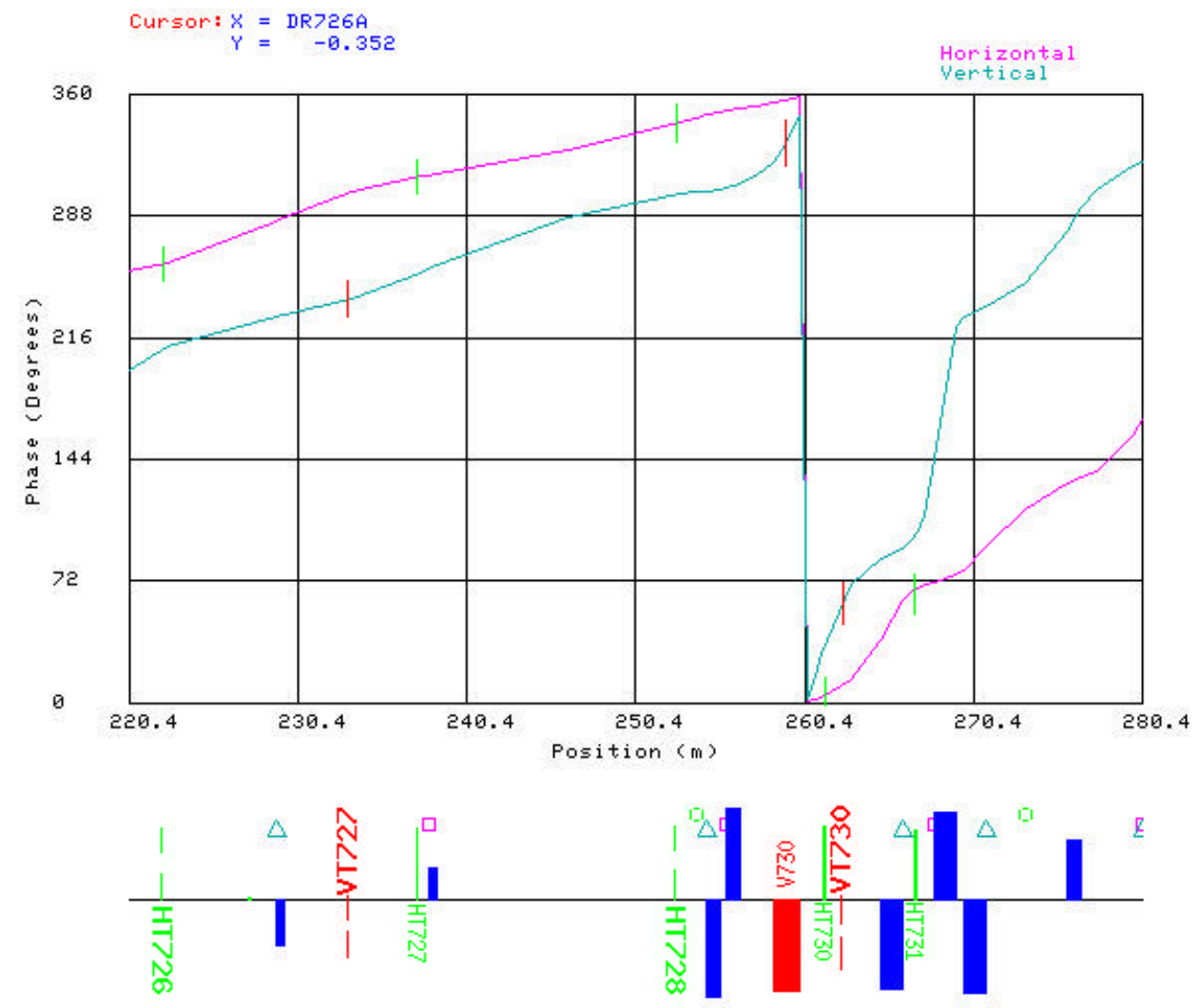

For control of the beam position and angle at the injection point, the trims would optimally be placed at intervals of $90^{\circ}$. The existing trim positions and the proposed trims are given in the following tables organized by distance (and phase advance) from the end of the AP2 line.

\begin{tabular}{|c|c|c|l|}
\hline & Phase Advance (deg.) & Distance (m) & \multicolumn{1}{|c|}{ Comment } \\
\hline VT730 & 252 & 17.3 & New - between HT730 and Q731 -- position control \\
\hline V730 & 353 & 21.1 & Exists - angle control \\
\hline VT727 & 443 & 46.8 & New - between Q727 and HT727 -- position control \\
\hline
\end{tabular}

Obviously, the injection septum also gives angle control. Unfortunately Q732 and Q733 are at the first place, phase advance of $90^{\circ}$, where one would like to establish position control.

Horizontally, HT731 seems to be placed well for controlling the position at injection. HT730 is not placed well for angle control; a better place would be upstream of the vertical bend (HT728). One would not want to move HT730 since the new combination of trims could also be used to create a bump 
through the vertical bend. However, moving HT727 to HT726 position could be considered instead of just adding HT726.

\begin{tabular}{|l|c|c|l|}
\hline & Phase Advance (deg.) & Distance $(\mathrm{m})$ & \multicolumn{1}{c|}{ Comment } \\
\hline HT731 & 97 & 13.3 & Exists - position control \\
\hline HT730 & 165 & 19.4 & Exists \\
\hline HT728 & 180 & 27.4 & New - just upstream of SEM728 - angle control \\
\hline HT727 & 212 & 42.5 & Exists \\
\hline HT726 & 266 & 57.7 & New - just downstream of Q726 - position control \\
\hline
\end{tabular}

In summary of the injection region, four new trims appear to give the control desired at the injection point according to the model. Three new trims could be used and HT727 could be left where it currently hangs or could be moved to HT726.

\section{Vertical Bump at the Left Bend}

Two new trims in addition to an existing trim can easily do a position bump through the left bend. The existing trim VT711 can be used but causes an excursion the opposite direction prior to the left bend bump; it was thought this is not desirable. One new trim is needed before the left bend: VT715 --just upstream of BPMH716. The second trim would be placed early in the left bend: VT717 (downstream of Q717), VT718 (between B3 and B4), or VT719 (between SEM719 and 719 collimators). The latter would possibly require moving the SEM and collimator can to make enough room. VT719 will be necessary to make an angle bump through the left bend. Below is a P143 display of a position bump using fictitious VT719 as the middle trim.
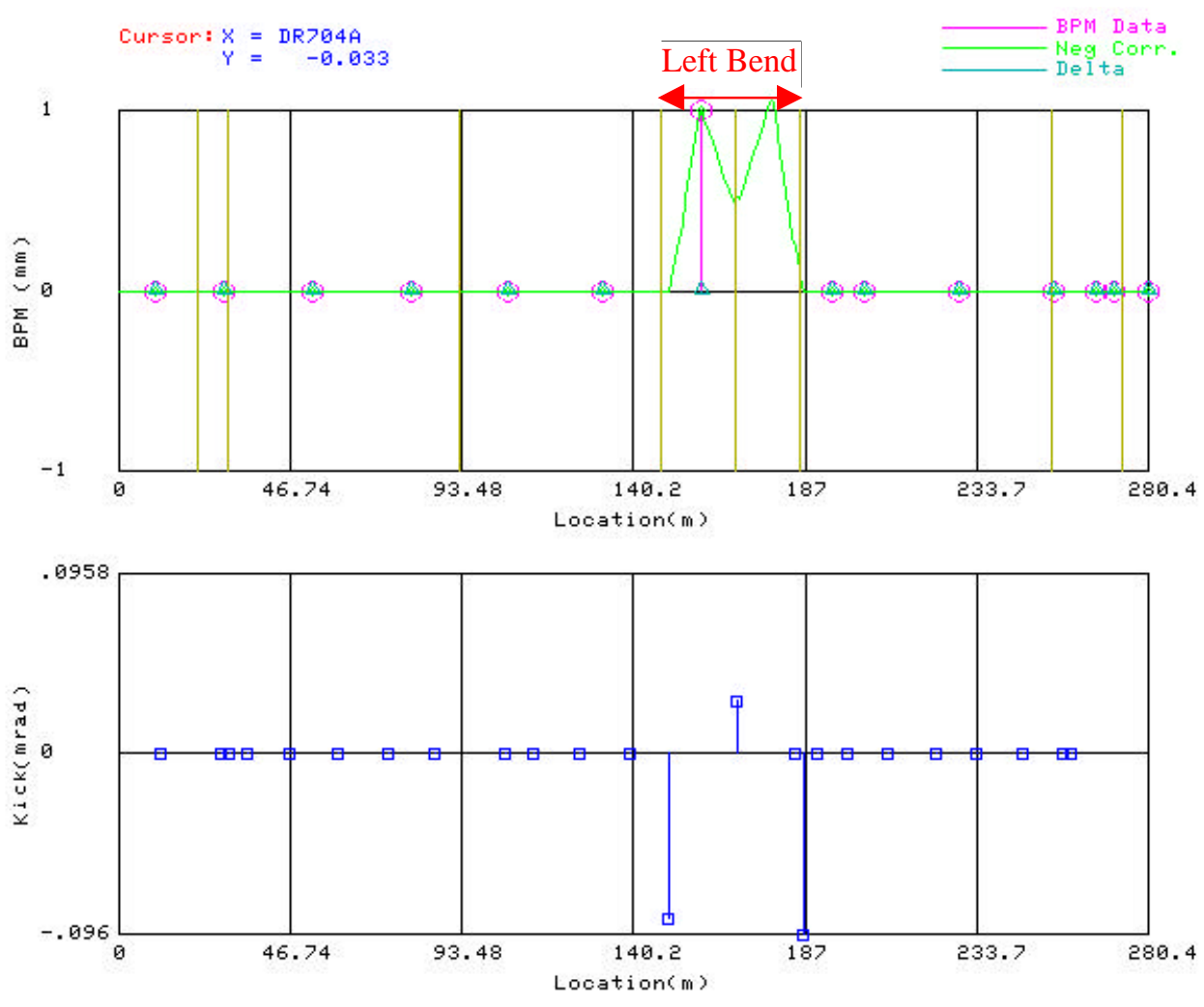